\title{
Work transition tips: Inclusion for workers with hearing loss: Actions for work practice professionals
}

\author{
L. Shaw ${ }^{\mathrm{a}}$, M.B. Jennings ${ }^{\mathrm{b}}$ and S.E. Kramer ${ }^{\mathrm{c}, \mathrm{d}}$

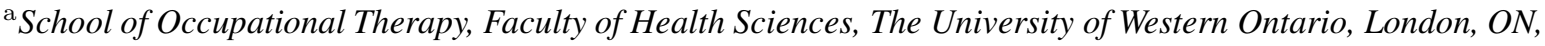 \\ Canada \\ ${ }^{\mathrm{b}}$ National Centre of Audiology, Faculty of Health Sciences, Western University, London, ON, Canada \\ ${ }^{c}$ VU University Medical Center, Department of ENT/Audiology, Amsterdam, the Netherlands \\ ${ }^{\mathrm{d}} \mathrm{EMGO}+$ Institute for Health and Care Research, Amsterdam, the Netherlands
}

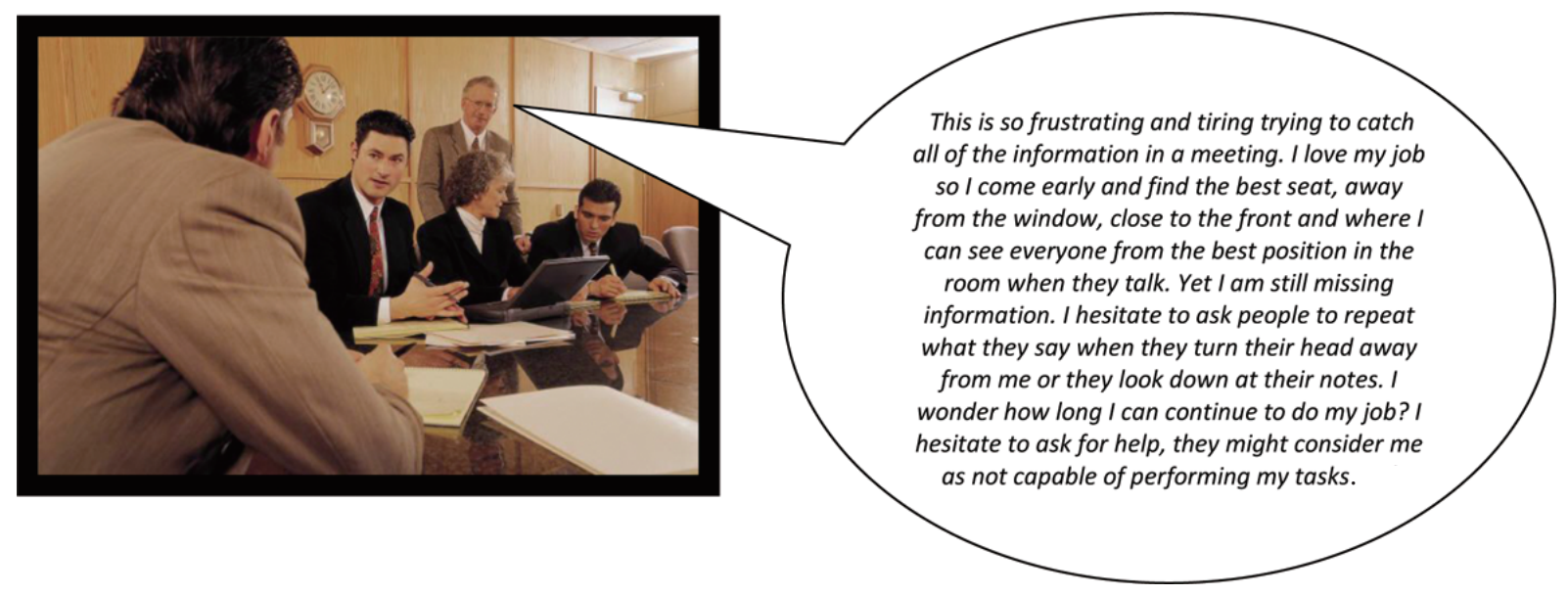

The global prevalence of adult hearing loss was estimated to be 538 million [1]. In the United States 20 million workers experience hearing loss [2]. Yet hearing loss at work is often unnoticed, underreported, and unaddressed. The hidden nature or invisibility of hearing loss can lead to overlooked work disparities. For instance, workers with hearing loss either withdraw early from work due to lack of accommodation or work longer, out of necessity, than other workers. They also experience lower work productivity (presenteeism) due to fatigue and/or can be mistaken for a worker who is disengaged in the workplace when in fact they purposefully limit participation in social events or teamwork to manage the stress associated with communicating in group settings. Recently, how- ever, the World Health Organization has played a role in raising hearing loss as a global chronic health issue of concern [3]. The WHO is advocating for the advancement of competencies in the health care workforce to enhance the health, wellbeing, participation and inclusion of persons with hearing loss in society.

This global call to attention on the burden of hearing loss presents a key leadership opportunity for work practice professionals to promote 'hearing at work'. The time is right for a renewed emphasis on evidence-informed wellness and rehabilitation approaches to prevent disability and marginalization of workers with hearing loss. This tip sheet offers insights into how work practice professionals can inform change. Knowledge innovations in this special 
Work practice professionals can facilitate 'hearing at work'

-Conduct an environmental scan of services and resource needs for workers with hearing loss and employers

-Identify hearing accessibility issues; use the Universal Design for Hearing guide

-Increase awareness of hearing demands in the workplace work using C-HearD tools

-Identify sector specific needs

-Identify groups of workers with hearing loss who are at risk for work disruption or work loss

-Identify groups of workers (by age, gender, etc,) marginalized from work due to hearing loss

-Educate organizations on compliance with legislation or inclusion policies

Establish Networks with key stakeholders to provide leadership in their communities that can support innovations in 'hearing at work':

- Create a community of practice

-Increase access to information on hearing loss

-Write an article in local newspaper

- Contribute to a blog to raise awareness

-Consider the expertise needed from other
Accessibility professionals can assist with technology Audiologists can offer HATs AR and UDH assessment Psychologists and other mental health professionals can provide coping strategies

Occupational therapists can support accommodation plans

Local business representatives can share best practices

Consumer organizations can provide education and support

Social workers can support access to funding

Occupational physicians can implement hearing surveillance

Primary Care Physicians can make referrals to specialists

Architects and interior designers can support inclusive

design in the built environment

Partner with employers and worker representatives to develop and/or disseminate information on:

- 'Hearing and communication' accommodation and accessibility plans for workers and customers

- Recruitment plans to hire persons with hearing loss

- Ongoing access to accommodation supports to support retention

-Disclosure strategies

-Co-worker training

-Awareness-raising of the benefits of hearing and cost benefits of proactive planning

- Universal Design Guidelines for Hearing principles

-Environments that support hearing

-Prevention of hidden consequences of managing hearing loss (stress, fatigue, continuous concern, mental distress)

-Psychosocial dynamics of hearing loss and importance to rehabilitation success

- Complexity of hearing function (worker characteristics \& the work environment)

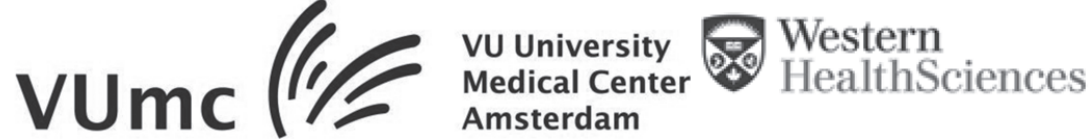

issue, along with the WHO suggested framework for addressing chronic conditions are used to posit steps that can enhance optimal hearing for all people in the workplace. Moving knowledge into action can provide workers, employers, and policy makers with integrated information that can support work participation of workers with hearing loss at work.

\section{References}

[1] S. Kochkin, Prevalence of hearing loss, Better Hearing Institute, retrieved on July 10, 2012 from http://www.betterhearing. org/hearing_loss/prevalence_of_hearing_loss/index.cfm.

[2] Stevens, G., Flaxman, S., Brunskill, E., Mascarenhas, M., Mathers, C. D., \& Finucane, M. (2011). Global and regional hearing impairment prevalence: An analysis of 42 studies in 29 countries. Eur J Public Health.

[3] Preparing a Health Care Workforce for the 21st Century: The Challenge of Chronic Conditions. WHO report. www.who.int/ chp/knowledge/publications/workforce_report/en/ - 22k.

For further information please contact leshaw @uwo. 